\title{
THE IMAGE OF $G$ BORDISM IN $Z_{2}$ BORDISM
}

\author{
R. PAUL BEEM
}

\begin{abstract}
For finite cyclic groups $G$ of even order, the image of unoriented $G$ bordism in $Z / 2 Z$ bordism and the kernel of the extension homomorphism from $Z / 2 Z$ to $G$ bordism depend only on whether or not the order of $G$ is divisible by four. If so, then these sets are equal and are equal to the image of circle bordism in $Z / 2 Z$ bordism and the kernel of extension to circle bordism. If not, then extension is a monomorphism and restriction is an epimorphism.
\end{abstract}

The purpose of this note is to show that for finite cyclic groups $G$ of even order the image of unoriented $G$ bordism in $Z / 2 Z$ bordism and the kernel of the extension homomorphism from $Z / 2 Z$ to $G$ bordism depend only on whether or not the order of $G$ is divisible by four. If so, then these sets are equal and are equal to the image of circle bordism in $Z / 2 Z$ bordism and the kernel of extension to circle bordism. If not, then extension is a monomorphism and restriction is an epimorphism.

We will use the definitions and notational conventions of [2]. Recall from [1] that there is an action, defined via the twisted product of $G$ spaces,

$$
N_{*}(G ; \text { Free }) \otimes N_{*}\left(G ; \mathscr{F}, \mathscr{F}^{\prime}\right) \rightarrow N_{*}\left(G ; \mathscr{F}, \mathscr{F}^{\prime}\right),
$$

which makes $N_{*}\left(G ; \mathscr{F}, \mathscr{F}^{\prime}\right)$ into an algebra over the Hopf algebra $N_{*}(G$; Free). The degree one endomorphism of $N_{*}\left(Z_{2}\right.$; All) given by twisting with the class of the circle with antipodal involution is a derivation and will be denoted by $\boldsymbol{\theta}$. In [1] it was shown that the kernel of this derivation is precisely the image of circle bordism.

TheOREM. Suppose $Z_{2} \subset G \subset S^{1}, G$ a finite group. If $Z_{4} \subset G$, then the following are equal in $N_{*}\left(Z_{2} ; \mathrm{All}\right)$ :

(A) kernel of extension e $(G): N_{*}\left(Z_{2} ; \mathrm{All}\right) \rightarrow N_{*}(G$; All $)$;

(B) kernel of extension e $\left(S^{1}\right): N_{*}\left(Z_{2}\right.$; All $) \rightarrow N_{*}\left(S^{1}\right.$; All $)$;

(C) kernel of $\theta: N_{*}\left(Z_{2} ;\right.$ All $) \rightarrow N_{*}\left(Z_{2} ;\right.$ All $)$;

(D) image of restriction $r\left(S^{1}\right): N_{*}\left(S^{1} ;\right.$ All $) \rightarrow N_{*}\left(Z_{2} ;\right.$ All $)$;

(E) image of restriction $r(G): N_{*}(G ; \mathrm{All}) \rightarrow N_{*}\left(Z_{2} ; \mathrm{All}\right)$.

Proof. Clearly A $\subset \mathrm{B}$ and D $\subset \mathrm{E}$. Since $\left(\left(S^{1} \times M\right) /(-1 \times T), 1 \times T\right)=$ $r\left(S^{1}\right) \circ e\left(S^{1}\right)(M, T)$, where $T$ denotes an involution on $M, \mathrm{~B} \subset \mathrm{C}$. C $=\mathrm{D}$ is shown in [1].

Received by the editors April 27, 1977 and, in revised form, June 2, 1977.

AMS (MOS) subject classifications (1970). Primary 57D85.

Key words and phrases. Equivariant bordism. 
Suppose $x$ is in $N_{*}\left(G\right.$; All). Then $e(G) \circ r(G)(x)=\left(G / Z_{2}, G\right) x$; see [2]. Since $G / Z_{2}$ has even order, $\left(G / Z_{2}, G\right)$ bounds in $N_{0}(G$; All $)$, so that $\left(G / Z_{2}, G\right) x$ bounds in $N_{*}(G$; All). See [2, p. 68]. Therefore E $\subset$ A. This finishes the proof.

If $G=Z_{2 n}$, where $n$ is odd, then $e(G)(M, T)=\left(M \times Z_{n}\right.$, $\left.\phi\right)$, where $\phi$ : $G \times\left(M \times Z_{n}\right) \rightarrow\left(M \times Z_{n}\right)$ is given by

$$
\begin{aligned}
& \phi\left(t,\left(m,\left(t^{2}\right)^{p}\right)\right)=\left(m,\left(t^{2}\right)^{p+1}\right), \quad p<n-1, \\
& =\left(T m, t^{2 n}\right), \quad p=n-1,
\end{aligned}
$$

where $t$ generates $Z_{2 n}$. Restricting this to a $Z_{2}$ action gives $n$ copies of $(M, T)$ which is bordant to $(M, T)$. Hence, $e(G)$ is monic and $r(G)$ is epic.

\section{REFERENCES}

1. R. P. Beem, On the action of free $G$ bordism on $G$ bordism, Duke Math. J. 42 (1975), 297-305.

2. R. E. Stong, Unoriented bordism and actions of finite groups, Mem Amer. Math. Soc.,no. 103, 1970.

Department of Mathematics, Indiana University, South Bend, Indiana 46615 